\title{
4-twist helix snake to maintain polarization in multi-GeV proton rings
}

\author{
F. Antoulinakis, Y. Chen, A. Dutton, E. Rossi De La Fuente, S. Haupert, E. A. Ljungman, \\ P. D. Myers, J. K. Thompson, A. Tai, C. A. Aidala, E. D. Courant, ${ }^{*}$ A. D. Krisch, M. A. Leonova, \\ W. Lorenzon, R. S. Raymond, D. W. Sivers, ${ }^{\dagger}$ V. K. Wong, and T. Yang \\ Spin Physics Center, University of Michigan, Ann Arbor, Michigan 48109-1040, USA
}

Y. S. Derbenev and V. S. Morozov

Thomas Jefferson National Accelerator Facility, Newport News, Virginia 23606, USA

A. M. Kondratenko

Science and Technique Lab "Zaryad", Akademika Lavrentieva prospect 6/1, off. 33, 630090 Novosibirsk, Russia

(Received 18 May 2017; published 27 September 2017)

\begin{abstract}
Solenoid Siberian snakes have successfully maintained polarization in particle rings below $1 \mathrm{GeV}$, but never in multi-GeV rings, because the spin rotation by a solenoid is inversely proportional to the beam momentum. High energy rings, such as Brookhaven's $255 \mathrm{GeV}$ Relativistic Heavy Ion Collider (RHIC), use only odd multiples of pairs of transverse B-field Siberian snakes directly opposite each other. When it became impractical to use a pair of Siberian Snakes in Fermilab's $120 \mathrm{GeV} / \mathrm{c}$ Main Injector, we searched for a new type of single Siberian snake that could overcome all depolarizing resonances in the 8.9-120 GeV/c range. We found that a snake made of one 4-twist helix and 2 dipoles could maintain the polarization. This snake design could solve the long-standing problem of significant polarization loss during acceleration of polarized protons from a few $\mathrm{GeV}$ to tens of $\mathrm{GeV}$, such as in the AGS, before injecting them into multi-hundred $\mathrm{GeV}$ rings, such as RHIC.
\end{abstract}

DOI: 10.1103/PhysRevAccelBeams.20.091003

To study the strong interaction's spin dependence with polarized proton beams [1] (see [2] for more references) one must preserve and control the polarization during acceleration and storage in rings. This is made difficult by many depolarizing spin resonances. In 1977 Derbenev and Kondratenko [3,4] proposed a clever way to overcome all depolarizing resonances by introducing a special sequence of magnets that would rotate each proton's vertical spin component by $180^{\circ}$ while leaving the proton's orbital motion in the ring unchanged (optical transparency). Courant named this sequence of magnets a Siberian snake [5]. The snake concept was first tested on a polarized $100 \mathrm{MeV}$ proton beam at the Indiana University Cyclotron Facility Cooler ring in 1989 [6] using a solenoid Siberian snake with longitudinal magnetic field. More recently, two facilities have used solenoid Siberian snakes to achieve polarized electron beams, the Amsterdam Pulse Stretcher [7] and the MIT Bates Ring [8].

\footnotetext{
*Also at BNL.

Also at Portland Physics Institute.
}

Published by the American Physical Society under the terms of the Creative Commons Attribution 4.0 International license. Further distribution of this work must maintain attribution to the author(s) and the published article's title, journal citation, and DOI.
Solenoid Siberian snakes are thus useful at low energies, but are difficult above a few $\mathrm{GeV}$ due to the Lorentz contraction of a solenoid's $\int B \cdot d l$ needed for the $180^{\circ}$ spin rotation. Derbenev and Kondratenko [3,4] noted that for transverse magnetic fields the $\int B \cdot d l$ seen by the protons is almost invariant under the Lorentz transformation at high energies. Thus, commissioned studies of how to accelerate and maintain polarized proton beams at the 20 -on- $20 \mathrm{TeV}$ SSC [9], Fermilab's Tevatron [10], and DESY's HERA [11] all planned to use pairs of transverse-field Siberian snakes. More importantly, Brookhaven has fabricated and used pairs of 4T helical Siberian snakes in the RHIC rings to successfully accelerate, store, and collide $100-255 \mathrm{GeV}$ polarized protons [12].

At energies below a few tens of $\mathrm{GeV}$, however, the orbit excursions within the transverse snake become too large or the space requirements for pairs of transverse snakes within the ring become excessive. In this paper, we focus on finding a Siberian snake solution for rings with medium energies - too high for a single solenoid Siberian snake to be implemented and too low for pairs of transverse Siberian snakes to be practical.

Recent studies [13] inspired by the spin dynamical properties of a figure-8 ring [14] indicate that one can compensate the main spin perturbation harmonics in a highenergy ring using multiple pairs of snakes with parallel axes. The main advantages of this scheme are that all of the snakes 
are identical and one can use the longitudinal-axis snake design described in this paper. It is much simpler and cheaper than that of a conventional helical snake that has its spin axis at an angle to the beam. This opens the possibility of using our snake design to accelerate polarized beams to high energies such as those of an EIC, LHC, and even a future FCC.

In flat horizontal rings, a beam proton's spin precesses around the ring dipole magnets' vertical fields. The spin tune $\nu_{s}=G \gamma$ is the number of spin precessions during one turn around the ring, where $\gamma$ is a proton's Lorentz energy factor and $G=(g-2) / 2=1.792847$ is its gyromagnetic anomaly. Horizontal magnetic fields can perturb the proton's stable polarization creating depolarizing spin resonances [15-19], which occur whenever $\nu_{s}=l \nu_{x}+m \nu_{y}+n$, where $l, m$ and $n$ are integers; $\nu_{x}$ and $\nu_{y}$ are the horizontal and vertical betatron tunes, respectively. Imperfection spin resonances occur when $l=m=0$. Intrinsic spin resonances occur when either $l \neq 0$ or $m \neq 0$, or both; the sum $|l|+|m|$ defines each resonance's order.

To overcome these depolarizing spin resonances, a full snake must rotate the vertical spin component by exactly $180^{\circ}$. The snake must also be optically transparent, which means that, to the rest of the ring, it appears to be an empty space. Moreover, the excursions caused by the snake must fit well inside the beam's vacuum chamber. The stable spin direction is vertical in a ring with pairs of snakes exactly opposite each other, when each pair's spin rotation axes are at $90^{\circ}$. In a ring with one snake with longitudinal spin rotation axis, the proton's stable spin direction is in the horizontal plane, and it is longitudinal directly opposite the snake.

Snakes using multitwist helical dipoles were first discussed in [4] and then studied in more detail by Kondratenko [20] and Courant [21]. We calculated properties of snakes with 1-, 2-, 3-, and 4-twist helices that have almost exactly longitudinal spin rotation axes and need only one vertical dipole at each end to obtain optical transparency. The magnetic fields and the protons' resulting excursions and spin behavior in the multitwist helix were obtained by treating them as multiple 1-twist helices in series. We assumed 4-T helical dipoles for our snake design, since the technology for building such magnets has been demonstrated at RHIC [12].

We first used a simplified model of the helical field to demonstrate the main features of the proposed design. It ignores coupling and optical focusing introduced by the longitudinal component and nonuniformity of the transverse component of a realistic helical field. These effects are inversely proportional to beam momentum but may become significant below $10-20 \mathrm{GeV} / \mathrm{c}$. However, they may be compensated by the ring's global decoupling and betatron tune control systems and have no impact on the conclusions of this paper.

Analytic calculations of the beam excursions inside the snake and the spin rotation were possible only for a simplified case of paraxial beam approximation and no fringe fields for the magnets. These calculations [2] were done using simplified on-axis magnetic field for an ideal 1-twist helical dipole

$$
B_{x}=B_{0} \sin k z, \quad B_{y}=B_{0} \cos k z,
$$

where the coordinates are: $x$ (radial), $y$ (vertical), and $z$ (longitudinal); while $k=2 \pi / L$ is the wave number of the $B$-field, and $L$ is the length of one twist. Spin calculations were done using formulas from Kondratenko [20] and the matrix for a one-twist helix obtained by Syphers [22]. We varied the helix lengths to rotate the vertical spin component by exactly $180^{\circ}$ at $120 \mathrm{GeV} / \mathrm{c}$, the Fermilab's Main Injector top energy.

We then used excursion equations from Courant [23] to calculate the maximum excursions for each number of twists. For Fermilab's $120 \mathrm{GeV} / \mathrm{c}$ Main Injector, the beam excursions caused by a 1-twist helix at the $8.9 \mathrm{GeV} / \mathrm{c}$ injection energy were too large, but those caused by a 4-twist helix were acceptable. Note that, in going from a 1-twist to 4-twist helix, the maximum transverse excursions inside the helical snake decrease almost 4-fold (from 4.76 to $1.26 \mathrm{~mm}$ at $120 \mathrm{GeV} / \mathrm{c}$ and 64.18 to $16.55 \mathrm{~mm}$ at $8.9 \mathrm{GeV} / \mathrm{c})$, while the total snake length increases by only $52 \%$ (from 4.242 to $6.459 \mathrm{~m}$ ).

In order to understand a snake with more complicated fields we also did numerical tracking calculations in Python. At each point along proton's path we used the Lorentz force equation,

$$
\vec{F}=q \vec{v} \times \vec{B},
$$

and the Thomas-BMT equation [24,25],

$$
\frac{d \vec{S}}{d t}=-\frac{q}{\gamma m}\left[(1+G \gamma) \overrightarrow{B_{\perp}}+(1+G) \overrightarrow{B_{\|}}\right] \times \vec{S},
$$

to calculate the changes in proton's trajectory and spin, respectively. We initially used simplified magnetic fields from Eq. (1) with 780000 steps (0.03 picosec/step) and compared the results to the analytic calculations (Fig. 1).

We recently did ultraprecise numerical calculations using the Blewett-Chasman fields [26], which satisfy Maxwell's equations and give small deviations of the fields from Eq. (1) away from the axis. We also reoptimized the length of the helix from $5.653 \mathrm{~m}$ to $5.652874 \mathrm{~m}$ for these ultraprecise calculations using a 0.05 picosec time step. We solved Eqs. (2) and (3) using the Blewett-Chasman fields,

$$
\begin{aligned}
& B_{x}=-B_{0}\left[1+\frac{k^{2}}{8}\left(3 x^{2}+y^{2}\right)\right] \sin k z-\frac{k^{2}}{4} x y \cos k z \\
& B_{y}=-B_{0}\left[1+\frac{k^{2}}{8}\left(x^{2}+3 y^{2}\right)\right] \cos k z-\frac{k^{2}}{4} x y \cos k z \\
& B_{z}=-k B_{0}(x \cos k z+y \sin k z)\left[1+\frac{k^{2}}{8}\left(x^{2}+y^{2}\right)\right],
\end{aligned}
$$



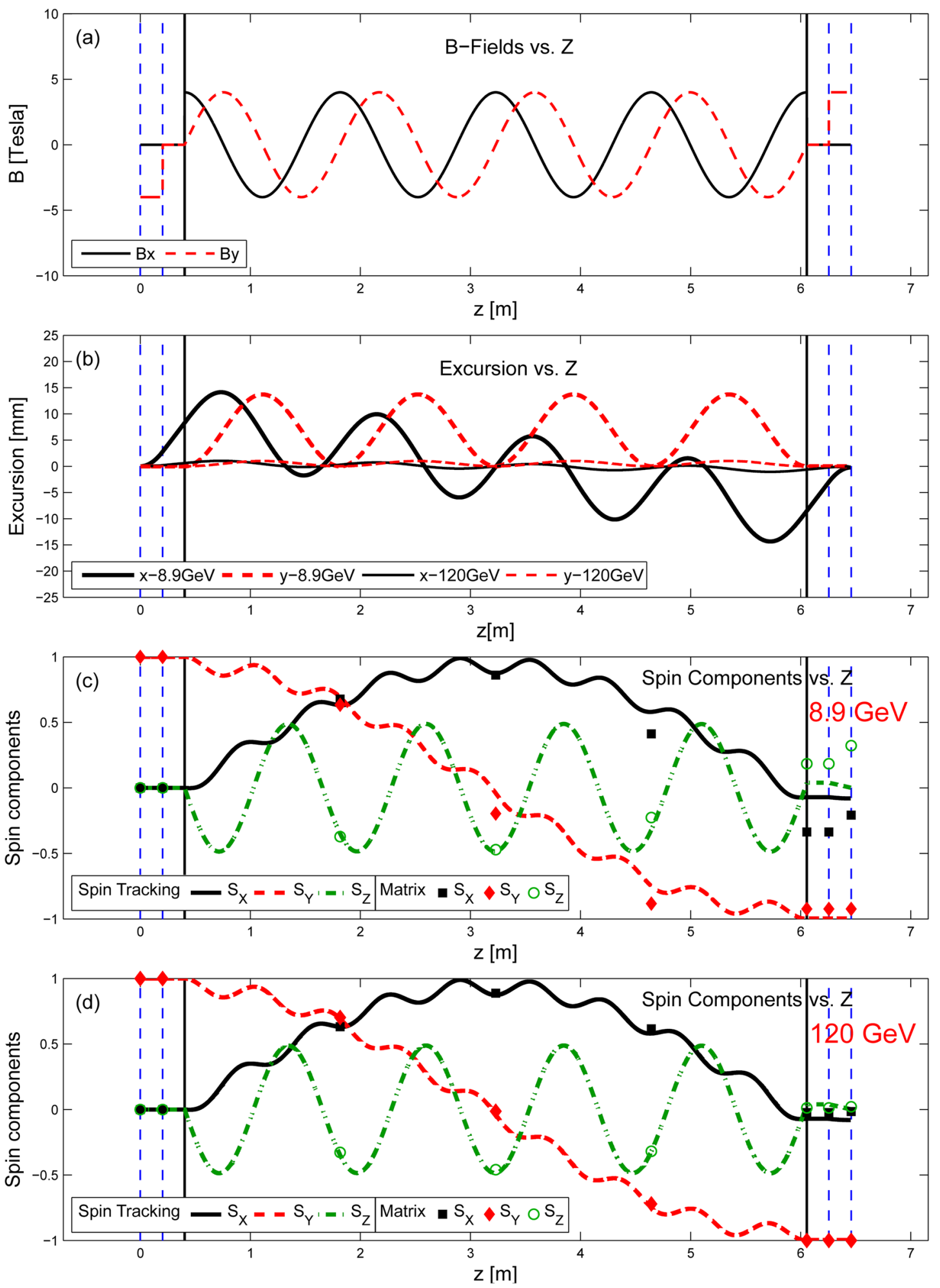

FIG. 1. (a) Horizontal and vertical field components for a Siberian Snake consisting of: 4-T-4-twist-5.653 m-long helical dipole, 4-T-0.203 m-long dipoles at each end and two $0.200 \mathrm{~m}$-long gaps [2]. Vertical lines show the helix's edges (solid black) and the dipoles' edges (dashed blue). In the graphs, the curves are from a Python-based spin and excursion tracking program, the symbols are for analytic matrix calculations. (b) Horizontal and vertical orbit excursions. (c) Radial, vertical, and longitudinal spin components for a $8.9 \mathrm{GeV} / \mathrm{c}$ beam. (d) Spin components for a $120 \mathrm{GeV} / \mathrm{c}$ beam. Note that while numerical calculations can give us spin orientation anywhere inside the snake, the matrix calculations are only available for the end of each magnet. Note the much better agreement between our numerical and matrix calculation at $120 \mathrm{GeV} / \mathrm{c}$ than at $8.9 \mathrm{GeV} / \mathrm{c}$. Fortunately a polarized proton beam spends almost all of its time at or near its final energy; the depolarization near $8.9 \mathrm{GeV} / \mathrm{c}$ should be much smaller. 
TABLE I. Spin components at the exit of the snake as a function of time-step used for numerical calculations using BlewettChasman fields, for a $120 \mathrm{GeV} / \mathrm{c}$ beam entering the snake onaxis with spin components $S_{X}, S_{Z}=0, S_{Y}=1$.

\begin{tabular}{lccr}
\hline \hline & \multicolumn{3}{c}{ Spin component } \\
\cline { 2 - 4 } $\begin{array}{l}\text { Time } \\
\text { picosec }\end{array}$ & $S_{X}$ & $S_{Y}$ & \multicolumn{1}{c}{$S_{Z}$} \\
\hline 0.15 & -0.0000285400 & -0.9999999998 & 0.0000119886 \\
0.14 & -0.0000134248 & -0.9999999991 & -0.0000452889 \\
0.13 & -0.0000225569 & -0.9999999998 & -0.0000166101 \\
0.12 & -0.0000003435 & -1.0000000002 & -0.0000007493 \\
0.11 & -0.0000139847 & -1.0000000001 & -0.0000004871 \\
0.10 & -0.0000132516 & -0.9999999996 & -0.0000289624 \\
0.09 & -0.0000191655 & -0.9999999999 & 0.0000057266 \\
0.08 & -0.0000002541 & -1.0000000001 & 0.0000073877 \\
0.07 & -0.0000129620 & -0.9999999999 & -0.0000025083 \\
0.06 & -0.0000130694 & -0.9999999999 & -0.0000127148 \\
0.05 & -0.0000129161 & -0.9999999999 & 0.0000015511 \\
0.04 & -0.0000087401 & -0.9999999999 & -0.0000005717 \\
0.03 & -0.0000097897 & -0.9999999999 & -0.0000005495 \\
0.02 & -0.0000108393 & -0.9999999999 & -0.0000005273 \\
0.01 & -0.0000076917 & -0.9999999999 & -0.0000005851 \\
0.008 & -0.0000070622 & -0.9999999999 & -0.0000005966 \\
0.005 & -0.0000071666 & -0.9999999999 & -0.0000006984 \\
0.002 & -0.0000072628 & -0.9999999999 & 0.0000002188 \\
\hline \hline
\end{tabular}

with various time-steps, as shown in Tables I and II and Figs. 2 and 3. We varied the time steps from 0.002 to 0.15 picosec, which is emphasized by vertical colored lines in the graphs, making them into quasilog plots.

TABLE II. Beam excursions and angles at the exit of the snake as a function of time-step used for numerical calculations using Blewett-Chasman fields, for a $120 \mathrm{GeV} / \mathrm{c}$ beam entering the snake on-axis.

\begin{tabular}{lcrrrr}
\hline \hline & \multicolumn{2}{c}{ Excursions $[\mu \mathrm{m}]$} & & \multicolumn{2}{c}{ Angle $[\mu \mathrm{rad}]$} \\
\cline { 2 - 3 } \cline { 5 - 6 } Time picosec & $X$ & $Y$ & & \multicolumn{1}{c}{$X$} & \multicolumn{1}{c}{$Y$} \\
\hline 0.15 & 0.2356 & -0.0046 & & -0.2248 & -0.0483 \\
0.14 & 0.8416 & -0.3011 & & -0.0697 & -0.1049 \\
0.13 & 0.6882 & 0.1969 & -0.0651 & -0.0116 \\
0.12 & 0.5269 & -0.7073 & -0.0600 & -0.2212 \\
0.11 & 0.5570 & -0.2876 & & -0.0550 & -0.0914 \\
0.10 & 1.3256 & 0.0752 & & 0.0496 & -0.1015 \\
0.09 & 0.6238 & -0.2106 & -0.0451 & -0.0415 \\
0.08 & 0.3978 & -0.8202 & -0.1199 & -0.2212 \\
0.07 & 1.0533 & -0.2915 & & 0.0347 & 0.1014 \\
0.06 & 0.6962 & -0.1505 & -0.0301 & 0.1014 \\
0.05 & 0.8515 & -0.3479 & -0.0751 & 0.1010 \\
0.04 & 0.7689 & -0.4488 & -0.0201 & -0.1413 \\
0.03 & 0.7992 & -0.4165 & & -0.0152 & -0.1313 \\
0.02 & 0.8294 & -0.3842 & & -0.0102 & 0.1214 \\
0.01 & 0.8597 & -0.4810 & -0.0052 & -0.1513 \\
0.008 & 0.8690 & -0.5004 & 0.0118 & -0.1573 \\
0.005 & 0.8143 & -0.4972 & 0.0027 & -0.1563 \\
0.002 & 0.8238 & -0.5052 & 0.0008 & -0.1553 \\
\hline \hline
\end{tabular}
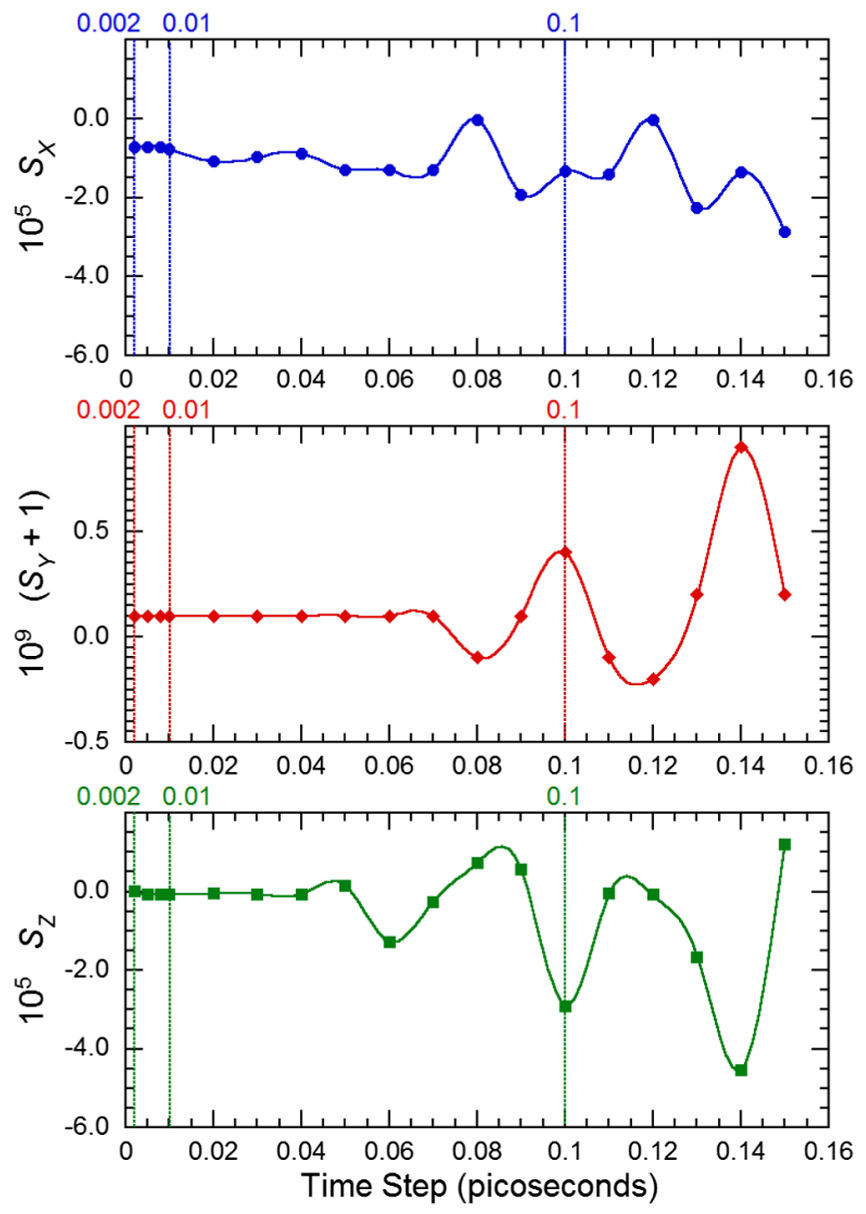

FIG. 2. Spin components at the exit of the snake as a function of time-step used for numerical calculations using Blewett-Chasman fields, for a $120 \mathrm{GeV} / \mathrm{c}$ beam entering the snake on-axis with spin components $S_{X}, S_{Z}=0$ and $S_{Y}=1$.

Table I and Fig. 2 show the results for spin components at the exit of the snake for a $120 \mathrm{GeV} / \mathrm{c}$ beam entering the snake on-axis. One can see that for the vertical spin component $\left(S_{Y}\right)$ the results begin to converge for time steps below 0.07 picosec. The component $S_{Y}$ is most important, since it must be rotated by exactly $180^{\circ}$ for a full snake. Table I and Fig. 2 show that we achieved $S_{Y}$ rotation by $180^{\circ}$ with very high precision; note the expanded vertical scales in Fig. 2. Note that $S_{Y}$ remained totally unchanged over the time-step range from 0.07 to 0.002 picosec, while the radial $\left(S_{X}\right)$ and longitudinal $\left(S_{Z}\right)$ spin components oscillate significantly. Also note that the $S_{Y}$ vertical scale is $10^{4}$ times smaller than the $S_{X}$ and $S_{Z}$ scales.

Table II and Fig. 3 show the results for the beam excursions and angles at the exit of the snake for a $120 \mathrm{GeV} / \mathrm{c}$ beam entering the snake on-axis. One can see that the results converge at time-steps below 0.005 picosec. The values of the beam excursions and angles at the snake exit are below $1 \mu \mathrm{m}$ and $0.16 \mu \mathrm{rad}$, respectively; this certainly indicates that the snake should look like a drift space to the rest of the ring. 


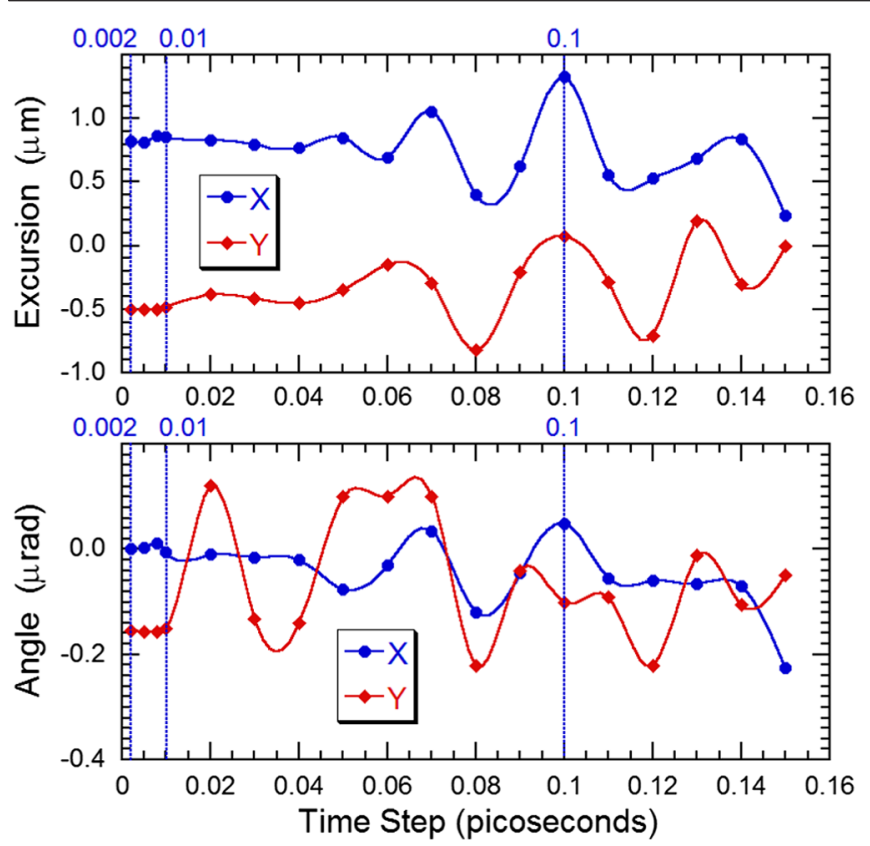

FIG. 3. Beam excursions and angles at the exit of the snake as a function of time-step used for numerical calculations using Blewett-Chasman fields, for a $120 \mathrm{GeV} / \mathrm{c}$ beam entering the snake on-axis.

In summary, by using an ultraprecise numerical Python spin tracking program, we have shown that one can rotate the vertical spin component by $180^{\circ}$ with very high precision. The 4-T transverse snake would contain a single $5.652874 \mathrm{~m}$-long 4-twist helix placed between two $0.203 \mathrm{~m}$-long dipoles, separated by gaps of $0.200 \mathrm{~m}$. This 4-twist helix snake should maintain the proton polarization in Fermilab's Main Injector during acceleration from 8.9 to $120 \mathrm{GeV} / \mathrm{c}$. By incorporating a multitwist helix into the core of a snake design, we were able to design a single snake practical for medium energies. It decreases the orbit excursions inside the snake, significantly, while increasing the space required for a single snake only slightly.

This compact single multitwist transverse snake design may provide the missing middle link in accelerating polarized beams from low energies, where solenoid snakes are practical, up to high energies, where multiple pairs of transverse snakes are most effective. In medium energy rings equipped with these compact snakes, one could inject low energy polarized protons, at a few $\mathrm{GeV}$, and accelerate them up to tens of, or even a hundred, $\mathrm{GeV}$ with negligible polarization loss. These highly polarized protons could then be injected into multi-hundred $\mathrm{GeV}$, or even $\mathrm{TeV}$, rings. Examples of medium energy rings include Japan's 30-50 GeV JPARC and Dubna's 12-25 GeV NICA deuteron-proton collider. Moreover, this compact single snake design might allow the $25 \mathrm{GeV}$ AGS to increase the polarization of its protons injected into the $255 \mathrm{GeV}$ RHIC, thus perhaps solving a problem that existed for many years [27]. Use of multiple identical snakes of our design in high-energy rings allows for polarized beam acceleration up to LHC and even FCC energies.

We thank I. Korbanis and H. B. White, Jr. for their advice on Fermilab's evolving Main Injector. We thank L.W. Jones for advice on this manuscript. We thank the University of Michigan for supporting this work.

[1] D. G. Crabb et al., Spin Dependence of High- $P \perp^{2}$ Elastic $p-p$ Scattering, Phys. Rev. Lett. 41, 1257 (1978).

[2] F. Antoulinakis et al., One 4-twist helix snake to maintain polarization in 8-120 GeV proton rings, arXiv:1309.1063.

[3] Y.S. Derbenev and A. M. Kondratenko, Proceedings of 10th International Conference on High Energy Accelerators 2, 70 (Protvino, 1977).

[4] Y.S. Derbenev and A. M. Kondratenko, AIP Conference Proceedings 51, 292 (AIP, NY, 1979).

[5] E. D. Courant, Acceleration and storage of polarized beams, AIP Conf. Proc. 42, 94 (1978).

[6] A. D. Krisch et al., First Siberian Snake Test, Phys. Rev. Lett. 63, 1137 (1989).

[7] G. Luijckx et al., in Proceedings of the Particle Accelerator Conference, Vancouver, BC, Canada, 1997 (IEEE, New York, 1997).

[8] T. Zwart et al., in Proceedings of the 19th Particle Accelerator Conference, Chicago, IL, 2001 (IEEE, Piscataway, NJ, 2001), p. 3597.

[9] R. D. Ruth et al., AIP Conference Proceedings (AIP, NY, 1986), Vol. 62.

[10] V. A. Anferov et al., Report No. UM HE 95-09, 1995.

[11] L. V. Alekseeva et al. (SPIN@HERA), Report No. UM-HE-96-20.

[12] I. Alekseev et al., Polarized proton collider at RHIC, Nucl. Instrum. Methods Phys. Res., Sect. A 499, 392 (2003); M. Bai et al., Polarized Proton Collision at $205 \mathrm{GeV}$ at RHIC, Phys. Rev. Lett. 96, 174801 (2006).

[13] S. Abeyratne et al., Science requirements and conceptual design for a polarized medium energy electronion collider at Jefferson Lab, arXiv:1209.0757; A. D. Kovalenko et al., in Proceedings of IPAC'15 (JACoW, Geneva, 2015), p. 2031.

[14] A. M. Kondratenko et al., Ion polarization scheme for MEIC, arXiv:1604.05632.

[15] M. Froissart and R. Stora, Depolarisation d'un faisceau de protons polarises dans un synchrotron, Nucl. Instrum. Methods 7, 297 (1960).

[16] T. Khoe et al., Acceleration of polarized protons to 8.5-GeV/c, Part. Accel. 6, 213 (1975).

[17] B. W. Montague, Polarized beams in high energy storage rings, Phys. Rep. 113, 1 (1984).

[18] F. Z. Khiari et al., Acceleration of polarized protons to 22 $\mathrm{GeV} / \mathrm{c}$ and the measurement of spin-spin effects in $p \uparrow+p \uparrow \rightarrow p+p$, Phys. Rev. D 39, 45 (1989).

[19] S. Y. Lee, Spin Dynamics and Snakes in Synchrotrons (World Scientific, Singapore, 1997), p. 26.

[20] A. M. Kondratenko, Doctoral thesis, (Budker) Institute of Nuclear Physics, Siberian Br. USSR Academy of Sciences, Novosibirsk, 1982 (in Russian). 
[21] E. D. Courant, Helical Siberian snakes, AIP Conf. Proc. 187, 1085 (1988).

[22] M. J. Syphers, BNL Internal Report No. AGS/RHIC/SN020, 1996.

[23] E. D. Courant, BNL Internal Report No. AGS/RHIC/SN010, 1996.

[24] L. H. Thomas, I. The kinematics of an electron with an axis, Philos. Mag. 3, 1 (1927).
[25] V. Bargmann, L.Michel, and V. L. Telegdi, Precession of the Polarization of Particles Moving in a Homogeneous Electromagnetic Field, Phys. Rev. Lett. 2, 435 (1959).

[26] J. P. Blewett and R. Chasman, Orbits and fields in the helical wiggler, J. Appl. Phys. 48, 2692 (1977).

[27] Increasing the AGS Polarization, edited by A. D. Krisch, M. T. Lin, and T. Roser, AIP Conf. Proc. (AIP, NY, 2002), Vol. 667. 